In Crescendo, 2019; 10(1): 71-88

Fecha de recepción: 23 de octubre del 2018

Fecha de aceptación: 23 de marzo del 2019

\title{
ESTUDIANTES FELICES: COMPETENCIAS DOCENTES VS SATISFACCIÓN ESTUDIANTIL
}

\author{
HAPPY STUDENTS: TEACHING COMPETENCIES VS STUDENT \\ SATISFACTION
}

\author{
Ulises Segura-Baron", \\ Clara Emilia Calderón-Perilla²
}

\section{RESUMEN}

El objetivo de esta investigación fue determinar relaciones entre las competencias docentes y la satisfacción estudiantil en la Universidad Piloto de Colombia a fin realizar una aproximación al concepto de felicidad de los estudiantes en la sección del Alto Magdalena. La investigación es de tipo descriptiva y correlacional, de diseño no experimental. Se identificó que existe una relación directa y significativa entre las competencias docentes y la satisfacción estudiantil; de forma similar de cada una de las dimensiones de las competencias docentes y la satisfacción de los estudiantes.

Se precisó que los estudiantes consideran que su nivel de satisfacción por la calidad de la enseñanza universitaria es alto; la dimensión más importante corresponde al dominio teórico y práctico de los docentes.

PALABRAS ClAVE: Competencias, eficiencia de la educación, calidad de la educación, estudiantes, educación superior

1 Universidad Piloto de Colombia.

2 Institución Educativa Manuel Elkin Patarrollo. 


\begin{abstract}
The objective of this research was to determine relationships between teaching competencies and student satisfaction at the Universidad Piloto de Colombia in order to make an approximation to the concept of happiness of students in the section of the Alto Magdalena. The research is descriptive and correlational, of non-experimental design. It was identified that there is a direct and significant relationship between teaching competencies and student satisfaction; similarly of each one of the dimensions of the teaching competences and the satisfaction of the students.

It was specified that students consider that their level of satisfaction with the quality of university education is high; the most important dimension corresponds to the theoretical and practical mastery of teachers.
\end{abstract}

KEY WORDS: Competencies, efficiency of education, quality of education, students, higher education.

\title{
INTRODUCCIÓN
}

El análisis de la calidad educativa en el marco del sistema educativo colombiano, presupone una gran complejidad de hechos entre los cuales las competencias docentes y la satisfacción de los estudiantes están involucrados de manera inmediata, estos dos aspectos, adquieren la caracteristica de objetos problémicos ante los que la universidad debe estar atenta para diagnosticar, evaluar y proponer acciones de mejoramiento continuo.

El realizar un análisis de las competencias docentes, que permitan relacionar sus resultados con la satisfacción de los estudiantes, orienta la implementaciòn de acciones para potenciar la calidad, la pertinencia y la equidad de los servicios educativos, no solo a nivel institucional, sino con gran utilidad a partir de la experiencia para universidades locales, regionales y nacionales.

Por ello, el propósito de esta investigación es contribuir al desarrollo de la Universidad Piloto de Colombia y que sus docentes mejoren su desarrollo profesional, comprometidos con su propio proceso de formación disciplinar y se generen procesos de innovación en sus prácticas pedagógicas (conscientes de que esto se debe reflejar en la satisfacción de los estudiantes de la universidad), el proceso investigativo inició con la exploración de espacios que dieran cuenta en tiempo presente de las características y propiedades del docente como tal y se elaboró a partir de ello un estudio de fortalecimiento de capacidades docentes para mejorar la satisfacción estudiantil en la Universidad Piloto de Colombia. 
Desde la dimensión teórica el estudio se apoyó en el análisis e interpretación de los conceptos de: capacidades docentes y satisfacción estudiantil, ampliamente definidos y discutidos en diferentes trabajos de investigación del escenario pedagógico. Entre ellos se destacan, respectivamente: Fernández (2012), Sepúlveda y Sáez (2014); Alves y Raposo (2004), Cortada y Woods (1995), Olave (Olaves, 2009) Bracho de Pereira (Bracho de Pereira, 2014), Alves y Raposo (2004), Capelleras y Venecia Una aproximación a la felicidad de los estudiantes.

Para orientar el proceso se consideró la afirmación de Osterhoff (2015): "dibujar y saborear las aguas más puras desde la misma primavera de las obras de Aristóteles ( Nunc ergo O iuvenes ex Aristotelico opere, ceu ex proprio fonte purissimas haurite, delibateque aquas )", (Oosterhoff, 2015, pág. parrafo 10) lo que aspiraba lefevre según Leonardo Bruni, sin citas escolásticas se placía de leer a Aristoteles. (Osterhoff, 2015).

Los estudiantes en lugar de iniciar con temas de marcada profundidad deben preferir una breve explicación de términos y conceptos sencillos con explicaciones simples pero concretas así podrían tener los espacios para disfrutar por ejemplo leer a Aristóteles afirma Oosterhoff, Richard J (Oosterhoff, 2015).

Es importante traer a colación lo expuesto por el premio nobel de económica el profesor Angus Deaton quien afirmó al respecto del problema de la desigualdad "No necesitamos abolir el capitalismo o nacionalizar selectivamente los medios de producción", "hay esperanza", (Deaton Angus;, 2018) aclara que la desigualdad no es lo mismo que la injusticia; pues afirma que algunas de los factores que impulsan la desigualdad son observados como justos, pero otros son profundamente injustos y son los que propician la ira y el rencor. (Deaton Angus;, 2018).

Así que, aunque parezca subjetivo el asunto es necesario ahondar en las aspiraciones creencias, cultura y expectativas de la gente, para el caso que se trae en cabeza de los estudiantes.

Investigadores del Instituto de Ciencias de la Felicidad de la Universidad Tecmilenio, demostraron que los estudiantes felices son los de mejores rendimientos académicos y que gozan de salud física saludable. Igualmente, la investigación reveló que el ambiente escolar positivo permite gozar de un mayor estado de felicidad de los estudiantes, y que el papel de los docentes es muy importante pues permite determinar las herramientas y especialidades necesarias para definir los propósitos de vida de los muchachos. (Mexico, 2015). 
El rector de la Universidad Tecmilenio, Doctor Héctor Escamilla afirmó que para obtener el mayor nivel de satisfacción de los estudiantes es necesario generar un clima laboral propicio, que coadyuve a potenciar al estudiante y que lo motive para la superación permanente. Además, señala que los estudiantes deben orientarse desde los primeros semestres a definir su proyecto de vida con lo cual el nivel de satisfacción será mayor. (Mexico, 2015).

Finalmente, y como primer corte teórico al tema, es preciso anotar lo expresado en su edición del 31 de enero del 2018 por el Espectador digital: en la U. de Yale, que ocupa el puesto 16 en el ranking de las instituciones más destacadas, cerca de 1.200 alumnos se matricularon en una clase que les ayuda a encontrar la felicidad. Es la que más asistentes ha tenido en los 316 años de existencia de esa universidad (Redacción educación el Espectador, 2018). Efectivamente como lo enuncia el diario, la docente es la profesora Laurie Santos, bióloga y psicóloga de la Universidad de Harvard y Ph.D en Psicología, de lo cual en New york time afirma puede ser una demostración de que los estudiantes quieren ser más felices, la profesora Santos dice que los estudiantes quieren su clase porque en el bachillerato ellos tuvieron que estudiar cosas que no era lo que ellos deseaban, que en sus clases los estudiantes deben cambiar patrones de comportamiento, deben abordar temas como proyectos de superación y que los estudiantes tienen otra visión de lo que es la felicidad, la cual ya no se puede entender cómo ganarse la lotería o cosas de ese estilo, sino por el contrario se buscan cambios reales en su estilos de vida. (Redacción educación el Espectador, 2018).

\section{A PROPÓSITO DE LAS COMPETENCIAS DOCENTES EN LA LABOR EDUCATIVA}

La labor educativa de los docentes ha sido sometida a grandes y profundas transformaciones, que van paralelas al desarrollo social de la humanidad, así que la formación docente exige características y condiciones para que su misión se concrete en la sociedad con base en la construcción de valores y cultura para garantizar la satisfacción de una sociedad, por cierto, afectada por los acontecimientos políticos y económicos de la época. En este orden de ideas, más que conocer y repetir contenidos, se debe propiciar la construcción de una sociedad fundamentada en criterios de equidad y responsabilidad.

Por lo que al abordar el estudio de las competencias docentes debe tenerse en cuenta la provisión de unas condiciones mínimas para el ejercicio de su pro- 
fesión, desde aspectos pedagógicos y didácticos hasta elementos éticos y humanísticos.

Sin duda la labor del docente en la Universidad comprende no solo acciones de enseñanza-aprendizaje. Hoy por hoy es indiscutible que la misión de la Universidad se dirige a interpretar las necesidades de la sociedad, de la empresa y del mismo Estado: es hacia esta relación que la actuación del docente debe orientarse: Universidad, Empresa, Estado y Sociedad.

Las anteriores apreciaciones entran en armonía con el preámbulo de la Conferencia Mundial sobre Educacion Superior promovido por la UNESCO en diciembre 2009: Las Nuevas Dinámicas de la Educación Superior y de la Investigación para el Cambio Social y el Desarrollo, en particular la que se trae a colacion:

Las instituciones de educación superior, a través de sus funciones de docencia, investigación y extensión, desarrolladas en contextos de autonomía institucional y libertad académica, deberían incrementar su mirada interdisciplinaria y promover el pensamiento crítico y la ciudadanía activa, lo cual contribuye al logro del desarrollo sustentable, la paz, el bienestar y el desarrollo, y los derechos humanos, incluyendo la equidad de género (2009, pág. 2).

\section{PRECISIÓN CONCEPTUAL DE COMPETENCIA}

Zabala y Arnau (2008) definen competencia como "La capacidad o habilidad de efectuar tareas o hacer frente a situaciones diversas de forma eficaz en un contexto determinado y para ello es necesario movilizar actitudes, habilidades y conocimientos al mismo tiempo y de forma interrelacionada". (Zabala \& Arnau, 2008, pág. 2).

En ese sentido, el término competencia indica, no tanto lo que uno posee, si no el modo en que uno actúa en situaciones concretas para realizar tareas de forma excelente. Por este motivo, las competencias tienen implícito el elemento contextual, referido al momento de aplicar estos saberes que la persona debe movilizar. La competencia siempre implica conocimientos interrelacionados con habilidades y actitudes.

Para Perrenoud (2007) el concepto de competencia representa "Una capacidad para movilizar varios recursos cognitivos para hacer frente a un tipo de situaciones". (Perrenoud, 2004, pág. 8).

Es prioritario transferir los elementos comunes del concepto de competencia, primero, a los procesos de prácticas educativas por competencias, enseguida, 
a los de identificación de competencias y por último, a la evaluación de competencias docentes.

Dentro de esta práctica, se concibe a un docente capaz de trabajar con estrategias y dispositivos de formación fundados en un modelo pedagógico que considere momentos de planeación auténtica, en la cual el profesor diseña situacionesproblema cercanas a la realidad del estudiante y orientadas a que este movilice sus recursos cognitivos para encontrar soluciones a las situaciones planteadas y, en ese proceso, tome decisiones y autorregule su aprendizaje.

\section{COMPETENCIAS DOCENTES}

Estas competencias docentes se expresan en desempeños genéricos que corresponden a las características de las funciones y acciones propias de prácticas docentes específicas, del quehacer cotidiano del docente que, para efectos prácticos de entendimiento, corresponden a la organización y ejercicio del trabajo escolar que el docente, de manera general, realiza en tres distintos momentos: antes (planeación macro y micro), durante (en el aula) y después.

Como lo infiere Pérez, 2011 planteando la necesidad de analizar las vías fundamentales por las que los seres humanos adquieren significados (aprenden), ya que esto constituye un referente obligado para el desarrollo competente del quehacer docente. Es decir, es necesario tener claro cómo aprende el alumno, ya que la tarea del profesor será la de apoyar estas formas de aprendizaje.

Ello supone un cambio radical de la función docente, desde las posturas de transmisión del conocimiento, hacia la valoración y apoyo a la evolución de los modelos que utilizan los alumnos para comprender los significados y proponer formas de actuación, que no descansan en la repetición memorística de datos o afirmaciones. Específicamente ¿Cuáles son los nuevos desempeños y competencias que el docente debe tener o desarrollar, para desenvolverse en una educación por competencias?

Por tanto, como lo afirma Rueda, "Uno de los primeros ejercicios en la selección de las competencias docentes podrá ser la discusión colegiada en cada uno de los contextos institucionales donde se adopte el enfoque por competencias." (Rueda Beltrán, 2009, pág. 8).

Una segunda ruta puede intentar identificar las competencias docentes empíricamente, mediante métodos más inductivos, empleando técnicas y estrategias que permitan caracterizar una práctica docente en particular y las competencias docentes presentes en ella, este referencial de competencias docentes. 
Según Perrenoud, 2004 hace una selección de competencias consideradas prioritarias porque son coherentes con el nuevo papel de los profesores, la evolución de la formación continua, las reformas de la formación inicial y las ambiciones de las políticas de la educación.

Igualmente, (Zabala \& Arnau, 2008) menciona entre otras: Planificar proceso de enseñanza y aprendizaje, manejar nuevas tecnologías, diseñar metodología y organizar actividades, comunicarse con los estudiantes, reflexionar e investigar sobre la enseñanza, identificarse con la institución y trabajar en equipo.

Se han considerado las siguientes dimensiones para el análisis de la variable "Competencias docentes", lo cual se sustenta en la Matriz de competencias del docente universitario en relación con la inclusión educativa Fernández,2012, págs. 20,21 tiene:

a) Experiencia en su disciplina: Debe ser de tal forma, que el profesional en educación piense y sienta su profesión desde el conocimiento, las destrezas y habilidades que conduzcan a procesos coherentes con los actos educativos. Cada reflexión en el aula expresa su bagaje y dominio pedagógico del contenido, desde lo práctico hasta lo científico.

b) Perspectivas pedagógicas: Implica que el docente considera al estudiante como el centro del proceso de enseñanza-aprendizaje; para lo cual, la docente incentiva al estudiante a mejorar sus conocimientos de forma frecuente.

c) Metodologías y técnicas didácticas: Un docente preparado para su campo de acción entra en contacto con el espacio propio del desarrollo de los procesos, particularmente en el contenido curricular. Esto se plasma concretamente en el currículo. En palabras de Celis y Gómez (2005), en la organización de la enseñanza:

El énfasis se centra en el despliegue y fortalecimiento de las capacidades e intereses del estudiante, como preparación (aprestamiento) para un futuro ocupacional incierto, imprevisible, cambiante, en el cual la capacidad de aprendizaje y recalificación continuos será más significativa que la acumulación de información y conocimientos especializados. (Gómez Campo \& Celis Giraldo, 2005, pág. 6).

d) Proceso de enseñanza - aprendizaje orientado a las necesidades laborales: Es el movimiento de la actividad cognoscitiva de los alumnos bajo la direc- 
ción del maestro, hacia el dominio de los conocimientos, las habilidades, los hábitos y la formación de una concepción científica del mundo.

e) Analizar y resolver problemas: Implica que el docente muestra disponibilidad y respeto para atender dudas y consultas respecto a la temática desarrollada.

\section{ESTUDIANTE SATISFECHO, ESTUDIANTE FELIZ}

Cortada y Woods (1995) definen en general la satisfacción como "La entrega de un producto o servicio que cumple o supera las expectativas o necesidades del cliente; y a los indicadores que determinan los niveles de satisfacción sobre el producto entregado o el servicio brindado". (Cortada, James W. Woods, John A, 1995).

Y con respecto a la universidad Flores precisa:

La categoría de clientes debe aplicarse a quienes tienen un contacto inmediato con la universidad en calidad de beneficiarios directos de su actividad, ya sea mediante una matrícula, un contrato, un acuerdo de patrocinio, la contratación de un titulado o la solicitud directa de cualquiera de sus servicios. De acuerdo con lo anterior, el beneficiario directo de los "productos" que ofrece la Universidad (certificaciones, títulos, grados, diplomados, etc.), que no son bienes tangibles, sino servicios de conocimiento, son los estudiantes y, por eso, a favor de ellos deben efectuarse todos los esfuerzos. (Flores, 2003, pág. 80).

\section{RESPECTO A LA MEDICIÓN DE SATISFACCIÓN ESTUDIANTIL}

Al examinar cómo se mide la satisfacción estudiantil se encuentra que tradicionalmente presenta grandes reparos entre otros Oliver (1997), citado por los profesores Alves y Raposo (2004) señala que:

"La medición de la satisfacción ha venido tradicionalmente consistiendo en poco más que en rellenar un simple cuestionario acerca de las diferentes características de un producto, ya se trate de un bien o un servicio, para así determinar cuáles son las que se tienen por causa o motivo de satisfacción o insatisfacción en relación con el mismo" (pág. 74).

Por otra parte, siguiendo la misma ruta Esta estrategia, basada en instrumentos tipo cuestionario se han venido elaborando para tratar de percibir la importancia y el grado de percepción que se tiene de dichas características. De la misma forma Elliot y Healy (2001), citados por Alves y Raposo (2004) refieren: 
Que, en la práctica, y en el caso concreto de la enseñanza universitaria, lo que los estudiantes suelen citar como importante en relación con su experiencia educativa, no suele ser, por lo general, indicativo de la satisfacción con dicha experiencia. (pág. 74).

En este orden de ideas, los autores afirman que los resultados basados en los estudios tradicionales escasamente muestran diversas características generales; no explican el por qué, el cómo y significación de las razones por las cuales se originan las condiciones de satisfacción o insatisfacción. Se tendría que abordar el tema a partir de plantear disyuntivas acerca de por qué una característica es un problema o un beneficio para el consumidor, buscando un resultado psicológico de esta tipología de conducta.

Capelleras y Veciana (2001), precisan que, dado que el reto para la educación superior se dirige hacia la calidad, se requiere precisar "Cuáles son el producto, las actividades clave y los clientes de la misma" (Capelleras \& Veciana, 2001). En este orden la EFQM (1995), citada por los autores, afirma que definitivamente el verdadero producto de la enseñanza son los conocimientos adquiridos y el plus añadido en términos de habilidades y experiencias académicas del que aprende, y citando a Álvarez y Rodríguez (1997:335) concreta que:

El producto sería el proceso de aprendizaje, tanto del alumno como del profesor. En este sentido, la calidad total implica la necesidad de que, no sólo el profesor sino también el estudiante, sea una parte activa en el diseño y en la creación y mejora continua de su propio proceso de aprendizaje. (pág. 7).

Acerca de las actividades institucionales que afectan a la calidad, precisan que se concluye de acuerdo con Capelleras y Veciana (2004) en su cita a Barnett (1992:113):

Las cuatro actividades que influyen en el aprendizaje del estudiante y su desarrollo educativo son: la calidad del método de enseñanza, del proceso de evaluación del alumno, la calidad de los cursos y del programa de desarrollo del profesorado. Estas actividades son comunes a toda institución de educación superior y son elementos para evaluar en una revisión de la actuación institucional. (pág. 7).

Así que, el cliente del servicio prestado en las universidades son los estudiantes, quienes constituyen los usuarios directos y principales del mismo; de donde se resalta que existe cada vez más la convicción de que es necesario contar 
con la opinión de los usuarios directos de la educación: los estudiantes. Ya que este es responsable de su propio aprendizaje y se constituye en coproductor activo de un servicio educativo.

\section{ASPECTOS METODOLÓGICOS}

La investigación fue de carácter descriptiva, los profesores Hernandez Sampieri, Baptista, \& Fernandez, 2010 afirman que esta requiere una especial rigurosidad en torno al conocimiento del área que se investigue, con una profundidad que permita desarrollar el proceso, aunando predicciones, observando el medio y con un conocimiento que, asociado a la correlación de las variables de estudio, logre finalmente explicar estas relaciones.

Diseño no experimental: Hernández Sampieri et al., manifiestan que este: "se realiza sin manipular deliberadamente variables" (Hernandez Sampieri, Baptista, \& Fernandez, 2010, pág. 189), dado que se trata de no hacerlas variar sino de observar, percibir y determinar sus movimientos para luego poder realizar análisis correspondientes de los cambios que ocurren en su ambiente natural.

Enfoque cualitativo: Retomando a Hernández Sampieri y otros, el enfoque cualitativo desde el cual se aborda el proceso investigativo se refiere a comprender y profundizar los fenómenos encontrados explorándolos desde la perspectiva de los participantes en su ambiente natural y en relación con el contexto. Así el enfoque cualitativo se seleccionó buscando comprender la perspectiva de los estudiantes acerca de los fenómenos que los rodean.

Metodológicamente se desarrolló a partir de la hipótesis general de que existe una relación directa y significativa entre la percepción sobre las competencias docentes y el nivel de satisfacción estudiantil (felicidad del estudiante).

Se procedió a la observación del medio y la correlación de las dos variables con sus movimientos e interacciones principales, sin perder de vista la comprensión de la perspectiva de los participantes: tanto estudiantes como profesores, acerca de los fenómenos objeto (satisfacción estudiantil y competencias docentes), profundizando en sus experiencias, perspectivas y percepciones de la realidad. No obstante, la población de la investigación fueron los 1304 estudiantes de la Seccional del Alto Magdalena, representados por 297 sujetos encuestados. 


\section{ANÁLISIS DE RESULTADOS}

\subsection{ANÁlISIS RESUMEN DE LA VARIABLE: COMPETENCIAS DOCENTES}

Tabla 1

COMPETENCIAS DOCENTES

\begin{tabular}{cccc}
\hline Variable & N & Media & Desv. Típ \\
\hline Competencias docentes & 297 & 3,7311 &, 69110 \\
\hline
\end{tabular}

\begin{tabular}{lcc}
\hline Nivel & Frecuencia & Porcentaje \\
\hline Bajo & 11 & $3,7 \%$ \\
Regular & 110 & $37,0 \%$ \\
Alto & 176 & $59,3 \%$ \\
\hline Total & 297 & $100,0 \%$ \\
\hline
\end{tabular}

\begin{tabular}{lcc}
\hline Dimensión & Media & Desv. típ. \\
\hline Experiencia en su disciplina & 3,7811 &, 82691 \\
Perspectivas pedagógicas & 3,7884 &, 75140 \\
Metodologías y técnicas didácticas & 3,7163 &, 72562 \\
Proceso de enseñanza-aprendizaje & & \\
orientado a las necesidades laborales & 3,6155 &, 78424 \\
Analizar y resolver problemas & 3,7616 &, 86212 \\
\hline
\end{tabular}

Fuente: Cuestionario "Competencias docentes".

\section{Análisis}

Los resultados encontrados sobre la variable "Competencias docentes", registrados en la tabla 1 , dieron un valor medio general de 3,73 y una desviación típica de 0,691, que permite concluir que los estudiantes de la Universidad Piloto de Colombia consideran que son de nivel adecuado o alto las competencias docentes que caracterizan a sus catedráticos; de forma específica se tiene que el $59,3 \%$ de los encuestados precisan que es de nivel alto las capacidades docentes, el 37,0\% precisa que es de nivel alto, y solamente el 3,7\% señala que es de nivel bajo. 
$\mathrm{Al}$ analizar por dimensión, en la figura 1 se tiene que los estudiantes consideran que el aspecto más destacado fue "Perspectivas pedagógicas", y el priorizado a mejorar la "Proceso de enseñanza - aprendizaje orientado a las necesidades laborales".

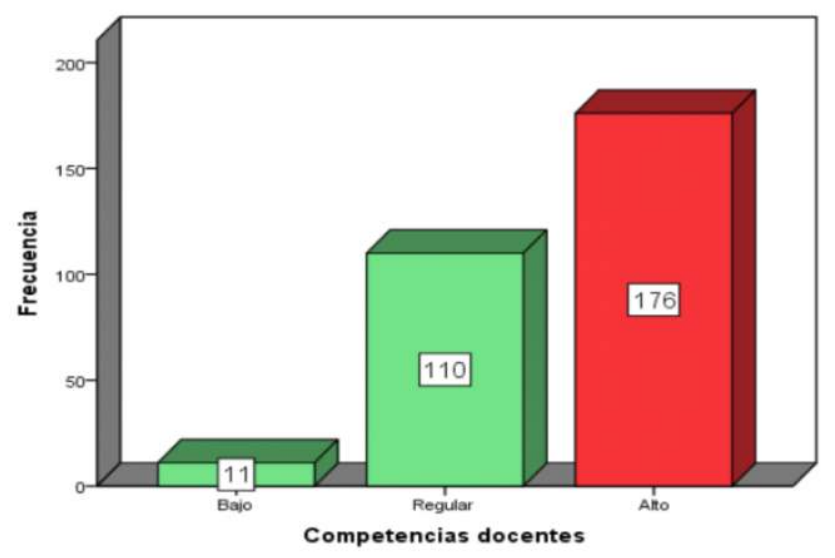

Figura 2. Competencias docentes.

Fuente: Cuestionario "Competencias docentes"

7.2. ANÁLISIS RESUMEN DE LA VARIABLE: SATISFACCIÓN ESTUDIANTIL

Tabla 2

SATISFACCIÓN ESTUDIANTIL

\begin{tabular}{cccc}
\hline Variable & N & Media & Desv. Típ \\
\hline Satisfacción estudiantil & 297 & 3,5997 &, 69270 \\
\hline
\end{tabular}

\begin{tabular}{lcc}
\hline Nivel & Frecuencia & Porcentaje \\
\hline Bajo & 17 & $5,7 \%$ \\
Regular & 136 & $45,8 \%$ \\
Alto & 144 & $48,5 \%$ \\
\hline Total & 297 & $100,0 \%$ \\
\hline
\end{tabular}


Tabla 2 (Cont.)

\begin{tabular}{lll}
\hline Dimensión & Media & Desv. típ. \\
\hline Instalaciones y equipamientos & 3,2071 &, 90376 \\
Interacción y clima laboral & 3,7082 &, 75695 \\
Dominio teórico y práctico de docente & 3,7367 &, 80373 \\
Organización de la enseñanza & 3,6465 &, 78508 \\
\hline
\end{tabular}

Fuente: Cuestionario "Satisfacción estudiantil".

\section{Análisis}

Los resultados encontrados sobre la variable "Satisfacción estudiantil", se muestran en la tabla 2 y dieron un valor medio general de 3,60 y una desviación típica de 0,693 , que permite concluir que los estudiantes de la Universidad Piloto de Colombia consideran que es de nivel adecuado o alto su nivel de satisfacción por la calidad de la enseñanza universitaria recibida en la entidad; de forma específica se tiene que el 48,5\% de los alumnos precisa que es elevado su nivel de satisfacción, el 45,8\% precisa que es de nivel regular, y solamente el 5,7\% señala que es de nivel bajo.

Igualmente, en la figura 2 se muestra como al analizar por dimensión, se tiene que los estudiantes consideran que el aspecto más destacado fue el "Dominio teórico y práctico del docente", y el priorizado a mejorar las "Instalaciones y equipamiento".

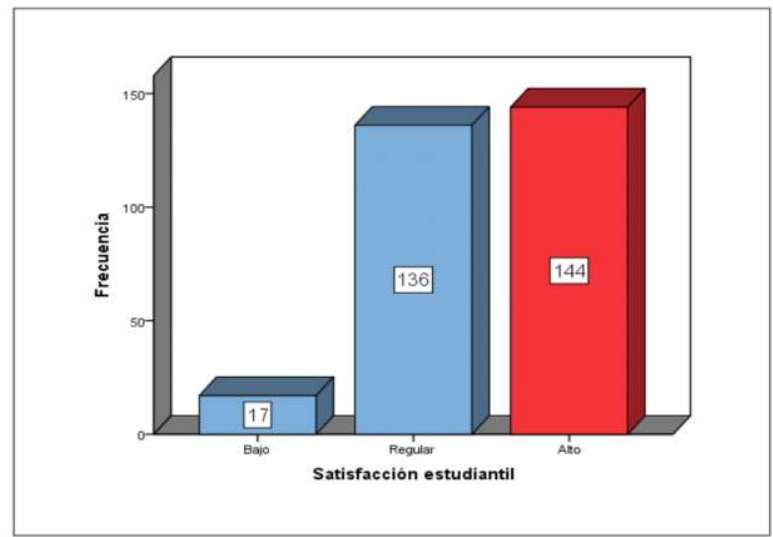

Figura 2. Satisfacción estudiantil.

Fuente: Cuestionario "Satisfacción estudiantil". 


\section{Contraste de la hipótesis general}

La hipótesis general planteada precisa que: "Existe una relación directa y significativa entre las competencias docentes y la satisfacción estudiantil en la Universidad Piloto de Colombia".

Para lo cual se aplicó el coeficiente de correlación de Rho de Spearman, cuyo reporte es:

$\mathrm{H}_{0}$ : No existe relación entre las variables.

$\mathrm{H}_{1}$ : Existe relación entre las variables.

Tabla 3

CONTRASTE DE LA HIPÓTESIS GENERAL

\begin{tabular}{|c|c|c|c|c|}
\hline & & & $\begin{array}{c}\text { Competencias } \\
\text { docentes }\end{array}$ & $\begin{array}{l}\text { Satisfacción } \\
\text { estudiantil }\end{array}$ \\
\hline \multirow{6}{*}{$\begin{array}{l}\text { Rho de } \\
\text { Spearman }\end{array}$} & \multirow{3}{*}{$\begin{array}{l}\text { Competencias } \\
\text { docentes }\end{array}$} & $\begin{array}{l}\text { Coeficiente de } \\
\text { correlación }\end{array}$ & 1,000 & ,580 \\
\hline & & Sig. (bilateral) & &, 000 \\
\hline & & $\mathrm{N}$ & 297 & 297 \\
\hline & \multirow{3}{*}{$\begin{array}{l}\text { Satisfacción } \\
\text { estudiantil }\end{array}$} & $\begin{array}{l}\text { Coeficiente de } \\
\text { correlación }\end{array}$ &, 580 & 1,000 \\
\hline & & Sig. (bilateral) &, 000 & \\
\hline & & $\mathrm{N}$ & 297 & 297 \\
\hline
\end{tabular}

Fuente: Los autores.

Puesto que el valor del $\mathrm{r}=0,580$ (valor de $\mathrm{p}=0,000$ ), lo cual permite concluir que como el valor de p es inferior al $5 \%$ de significancia, por tanto, existe una relación directa y significativa entre las competencias docentes y la satisfacción estudiantil en la Universidad Piloto de Colombia.

\section{DISCUSIÓN DE RESULTADOS}

En lo que respecta al comportamiento medio de la variable "Competencias docentes", se concluyó que los estudiantes de la Universidad Piloto de Colombia consideran que las competencias que caracterizan a sus catedráticos son de nivel adecuado; siendo la dimensión más destacada el "Perspectivas pedagógicas", y el priorizado a mejorar la "Proceso de enseñanza-aprendizaje orientado a las necesidades laborales". Siendo el análisis por dimensión: 
Sobre la dimensión "Conocimiento experto en su disciplina", se identificó que los estudiantes de la Universidad Piloto de Colombia consideran que el conocimiento experto del docente en su disciplina es adecuado; siendo lo más destacado el que el docente demuestra dominio de la materia enseñada, y el aspecto a mejorar es que el docente debería promover el uso de material bibliográfico complementario a lo enseñando en clases.

Sobre la dimensión "Perspectivas pedagógicas", se determinó que los estudiantes de la Universidad Piloto de Colombia consideran que el nivel de conocimiento de diversas perspectivas pedagógicas por parte de sus catedráticos es aceptable; siendo lo más destacado que el docente es respetuoso con cada uno de los estudiantes, y el aspecto a mejorar es que el docente debería dejar bien en claro los lineamientos generales para el aprendizaje.

Sobre la dimensión "Metodologías y técnicas didácticas", se concluyó que los estudiantes de la Universidad Piloto de Colombia consideran que el nivel de uso de diversas metodologías y técnicas didácticas que caracteriza a sus catedráticos es de nivel aceptable; siendo lo más destacado que la interacción entre los docentes y los estudiantes promueven el aprendizaje. El aspecto de mejora hace referencia a la organización del material didáctico de apoyo al desarrollo de clases.

Sobre la dimensión "Proceso de enseñanza - aprendizaje orientada a las necesidades laborales", se evidenció que los estudiantes de la Universidad Piloto de Colombia consideran que la capacidad del docente de orientar el proceso de enseñanza-aprendizaje centrado en el mercado laboral es de nivel aceptable; siendo lo más destacado que el estudiante percibe que existe una correspondencia entre los objetivos del curso y la evaluación aplicada, y el aspecto a mejorar es que el docente debería devolver los resultados de pruebas de forma oportuna.

Sobre la dimensión "Analizar y resolver los problemas", se concluyó que los estudiantes de la Universidad Piloto de Colombia consideran que la capacidad del docente de analizar y resolver los problemas es de nivel aceptable; siendo lo más destacado que el estudiante percibe que el docente se caracteriza por su ética profesional, y el aspecto a mejorar es que el docente debería recuperar las clases perdidas.

En lo que respecta al análisis del comportamiento medio de la variable "Satisfacción estudiantil”, se concluye que los estudiantes de la Universidad Piloto de Colombia consideran que su nivel de satisfacción por la calidad de la enseñanza universitaria recibida es adecuado; siendo la dimensión más destacada el "Dominio teórico y práctico del docente", y el priorizado a mejorar: las "Instalaciones y equipamiento”. Siendo el análisis por dimensión: 
Sobre la dimensión "Instalaciones y equipamientos", se concluye que los estudiantes de la Universidad Piloto de Colombia consideran que las características del equipamiento y las instalaciones de la entidad son de nivel regular; siendo el aspecto priorizado de mejora, el que las instalaciones de la universidad deberían ser más cómodas y acogedoras que impulsen el estudiar.

Sobre la dimensión "Interacción y clima laboral", se concluye que los estudiantes de la Universidad Piloto de Colombia consideran que el nivel de interacción y clima institucional que se da en la entidad es aceptable; siendo el aspecto más destacado que el estudiante considera que el docente fomenta con frecuencia la participación en clase, y el aspecto de mejora implica que el docente debería motivar más el revisar los apuntes de las clases previas.

Sobre la dimensión "Dominio teórico y práctico del docente", se concluye que los estudiantes de la Universidad Piloto de Colombia consideran que el nivel de dominio teórico y práctico del docente sobre el contenido de su asignatura es de nivel aceptable; siendo el aspecto más destacado que el estudiante considera que el docente tiene un nivel adecuado de dominio teórico de su asignatura, y el aspecto de mejora es que el docente debería promover el diálogo para poder tomar exámenes de recuperación.

Sobre la dimensión "Organización de la enseñanza", se concluye que los estudiantes de la Universidad Piloto de Colombia consideran que el nivel de organización de la enseñanza que caracteriza al docente es de nivel aceptable; siendo el aspecto más destacado que el estudiante considera que los horarios de clase son adecuados para la enseñanza, y el aspecto de mejora es que en el plan de estudios debería existir una mejor combinación entre la cantidad de asignaturas básicas y de especialidad.

Los resultados encontrados coinciden con el trabajo de investigación desarrollado por Olaves, 2009, puesto que ahí se logró concluir que los docentes logran aplicar métodos y estrategias que permiten lograr en sus alumnos aprendizajes significativos, tomando en cuenta la innovación y la creatividad, desempeñando diversos roles tanto dentro como fuera del aula, y desarrollando principalmente competencias como: ser creativo, abierto al cambio, dinámico, liderar, investigar, actualizarse.

También se resalta la coincidencia con los resultados encontrados por Bracho de Pereira, 2014, que resalta que los alumnos logran resaltar un alto nivel de 
sentido de pertenencia a la institución y el grupo de clases; que la infraestructura y el servicio prestado se encuentran dentro de las expectativas, y que existe satisfacción en cuanto al proceso educativo.

\section{CONCLUSIONES}

Se concluye que al existir una relación directa y significativa entre las competencias docentes y la satisfacción estudiantil; que los estudiantes consideran que las competencias pedagógicas de sus docentes son de nivel aceptable; y que su nivel de satisfacción por la calidad de la enseñanza universitaria recibida es alto. Así que los estudiantes presentan condiciones favorables para su felicidad.

Existe una relación directa y significativa entre la experiencia en su disciplina, perspectivas pedagógicas, metodologías y técnicas didácticas, el aprendizaje orientado a las necesidades laborales y la satisfacción estudiantil en la Universidad Piloto de Colombia. Se confirma las condiciones y aspiraciones de los estudiantes en torno a su felicidad.

\section{REFERENCIAS BIBLIOGRÁFICAS}

Alves H, \& Raposo, M. (2004). La medición de la satisfacción en la enseñanza universitaria: el ejemplo de la Universidad da Beira Interior. Revista Internacional de Marketing Público y No Lucrativo, 1(1), 73-88. Obtenido de:

https://www.academia.edu/22090261/La_medici\%C3\%B3n_de_la_satisfacci\%C3\%B3n_e n_la_ense\%C3\%B3anza_universitaria_El_ejemplo_de_la_universidade_da_beirainterior

Bracho de Pereira, Y. (2014). Análisis de satisfacción en los estudiantes de la escuela primaria nacional “José Ladislao Andara”. Punto Fijo. Venezuela.

Cano, H. (2005). Cómo mejorar las competencias de los docentes. Guía para la autoevaluación y el desarrollo de las competencias del profesorado. España: Barcelona Grao.

Capelleras, J.-L., \& Veciana, J. (2001). Calidad de Servicio en la Enseñanza Universitaria: Desarrollo y Validación de una Escala de Medida. Barcelona, España. Obtenido de https://ddd.uab.cat/pub/estudis/2001/hdl_2072_1032/UABDT01-4.pdf

Cortada, James W. Woods, John A. (1995). Encyclopedia of quality: terms and concepts. McGraw-Hill.

Deaton Angus;. (7 de Enero de 2018). El Espectador. Recuperado el 7 de 2 de 2018, de https://www.elespectador.com/economia/asi-funciona-la-desigualdad-segun-el-nobel-deeconomia-2015-articulo-732210?utm_source $=$ Whatsapp\&utm_medium =organic\&utm_ campaign $=$ Compartido-Mobile

Fernández, J. (2012). Capacidades y competencias docentes para la inclusión del alumnado en la educación superior. Red de revistas cieníificas de América Latina, el Caribe, España y Portugal, 8-24. 
Flores, J. (Julio de 2003). La satisfaccion estudiantil como indicador de la calidad en la educacion superior. Revistas de Investigación., 77-83. Obtenido de:

http://revistasinvestigacion.unmsm.edu.pe/index.php/educa/article/viewFile/8178/7131

Gómez Campo, V., \& Celis Giraldo, J. (2005). Factores de innovación curricular y académica. Revista Iberoamericana de Educación. Recuperado el 21 de 9 de 2018, de file://C:/Users/ ulises-segura/Downloads/773Gomez.PDF

Hernandez Sampieri, Baptista, \& Fernandez. (2010). Metodología de la Investigación. Mexico : Mcgraw-hill.

Mexico, U. (30 de Marzo de 2015). Universia. Obtenido de:

http://noticias.universia.net.mx/educacion/noticia/2015/03/30/1122429/estudiantesfelices-mejor-rendimiento-academico-alcanzan.html

Olaves, R. V. (2009). Competencias del docente de educación integral en el contexto de la realidad educativa venezolana. REDHECS: Revista electrónica de Humanidades, Educación y Comunicación Social,(7), 80-98. Obtenido de:

https://dialnet.unirioja.es/servlet/articulo? codigo $=3063108$

Oosterhoff, R. J. (10 de abril de 2015). The Stanford Encyclopedia of Philosophy. Recuperado el 7 de 2 de 2018, de https://plato.stanford.edu/entries/lefevre-etaples/

Padilla, J. E. (2008). La formación del docente universitario con miras al desarrollo humano. Educación y desarrollo social. Revista Educación y desarrollo social, 90-99. Obtenido de http://www.umng.edu.co/documents/63968/80131/RevNo1vol2.Art6.pdf

Pérez, Á. (2011). ¿Competencias o pensamiento práctico? La construcción de los significados de representación y acción. Ediciones Morata, S. L. Obtenido de:

https://sinectica.iteso.mx/index.php/SINECTICA/article/view/122

Perrenoud, P. (2004). Diez nuevas compentencias para enseñar. Grao. Obtenido de: https://www.uv.mx/dgdaie/files/2013/09/Philippe-Perrenoud-Diez-nuevas-competenciaspara-ensenar.pdf

Redacción educación el Espectador. (7 de 2 de 2018). El Espectador. Recuperado el 7 de 2 de 2018, de https://www.elespectador.com/noticias/educacion/felicidad-la-clase-mas-popular-enuna-de-las-mejores-universidades-del-mundo-articulo-736435

Rueda Beltrán, M. (2009). La evaluación del desempeño docente: consideraciones desde el enfoque por competencias. Revista electrónica de evaluación educativa. Obtenido de http://www.scielo.org.mx/scielo.php?script=sci_arttext \&pid=S1607-40412009 000200005

Sepúlveda, A. O. (2014). El docente universitario: capacidades pedagógicas para hacer clases, percepción de sus protagonistas. Revista de estudios y experiencias en educación., 67.

UNESCO. (2009). Las Nuevas Dinámicas de la Educación Superior y de la Investigación para el Cambio Social y el Desarrollo. Obtenido de:

http://www.me.gov.ar/spu/documentos/Declaracion_conferencia_Mundial_de_Educacion Superior_2009.pdf

Zabala, A., \& Arnau, L. (2008). 11 Ideas Claves como aprender y enseñar competencias. Recuperado el 21 de 9 de 2018, de:

https://www.cife.edu.mx:

https://www.cife.edu.mx/Biblioteca/public/Libros/7/documento\%2011\%20ideas\%20claves .pdf 